\title{
Knowledge Level Assessment in e-Learning Systems Using Machine Learning and User Activity Analysis
}

\author{
Nazeeh Ghatasheh \\ Department of Business Information Technology \\ The University of Jordan \\ Aqaba, 77110, Jordan
}

\begin{abstract}
Electronic Learning has been one of the foremost trends in education so far. Such importance draws the attention to an important shift in the educational paradigm. Due to the complexity of the evolving paradigm, the prospective dynamics of learning require an evolution of knowledge delivery and evaluation. This research work tries to put in hand a futuristic design of an autonomous and intelligent e-Learning system. In which machine learning and user activity analysis play the role of an automatic evaluator for the knowledge level. It is important to assess the knowledge level in order to adapt content presentation and to have more realistic evaluation of online learners. Several classification algorithms are applied to predict the knowledge level of the learners and the corresponding results are reported. Furthermore, this research proposes a modern design of a dynamic learning environment that goes along the most recent trends in e-Learning. The experimental results illustrate an overall performance superiority of a support vector machine model in evaluating the knowledge levels; having $\mathbf{9 8 . 6 \%}$ of correctly classified instances with $\mathbf{0 . 0 0 6 9}$ mean absolute error.
\end{abstract}

Keywords-Concept Maps; Multi-Class Classification; Machine Learning; Electronic Learning; Activity Analysis

\section{INTRODUCTION}

Incorporating the technology in the learning process is complex, but at the same time may shift the paradigm of learning and training. Authors in literature [1], [2] argue that eLearning offers a balance between the technology enablers and the acceptance issue. According to a twenty-year chronology of e-Learning evolution, Rosenberg in [1] made available an important road map for keeping the e-learning in a sustainable and continuous growth.

Automatic knowledge evaluation is an important component of online learning systems due to the nature of such systems, as the absence of direct contact between instructors and learners. On the other hand, content presentation needs to be tailored according to personal knowledge levels in some contexts. Furthermore, commonly used examinations and assignments may not reflect the actual knowledge level precisely in the context of automatic evaluation. Such issues regarding online learners evaluation drags the attention to a more comprehensive evaluation scheme. For example the time spent by online users in reading the content and the progress may give reasonable indications of the knowledge level.

e-Learning as a system of knowledge dissemination and evaluation has been assessed by various researchers [3]-[8]. Part of the assessment tackled the usability of such systems using machine learning [3], acceptance [4], the effect of student satisfaction on the learning process [5], and the integration of social networks [8]. It is clear that the dynamics of an eLearning system may affect the learning process in both the positive or negative directions.

Despite the bottlenecks and diversity of learners, it is still a promising trend that is expanding over time to shift towards electronic means of learning and evaluation [9]. Furthermore, mobile e-learning is expected to be a major trend according to the statistical study in [6] that investigated the behaviors for mobile learning.

Going further in developing futuristic e-learning platforms and approaches requires intensive analysis of more than an exam paper or in class assignment. Complex and wide spread learning activities ask for an intervention of artificial intelligence to reveal hidden or cluttered online activities. Data mining techniques represent an example of the computer intervention to address different e-Learning issues [10].

The behaviors of the learners in electronic learning systems are collected by different means in [6], for example the mobile device sensors, touch screen activity monitor, and mini keyboard input. Further collections of some behaviors are gathered and measured in [11], then a classification scheme is used to evaluate the users knowledge.

This research work tries to find the best algorithm among different machine learning approaches for classification, in order to recommend a suitable autonomous evaluator for the user's knowledge level in dynamic e-Learning systems. The outcomes of this research are expected to aid in building an efficient architecture of future dynamic e-Learning environments. Furthermore, it would illustrate different performance considerations of the classification approaches in the problem domain. The main objective in brief is to recommend an automatic evaluator of learners, which takes into account the user's activity beside the various forms of examinations.

This document is structured as follows: Section II highlights the related work the related work that employed machine learning in building evaluation systems. Section III presents a proposed architecture of an evaluation system in dynamic eLearning environments, Section IV describes the used data set and the evaluation metrics for the classification algorithms. The empirical results of the classification algorithms and the comparisons are discussed in Section V. Then concluding with a summary of the remarks and possible future work. 


\section{RELATED WORK}

The use of computer intelligence in user-adaptable eLearning environments has been addressed and examined by several researchers.

An interesting approach is presented in [11] for modeling the knowledge of e-Learning users under different domains. The approach is composed of a generic domain object model, user modeling, weight adjusting method, and a classification algorithm. The authors claim a successful implementation of a robust, domain independent and efficient approach that relies on Bayesian Network and Nearest Neighbor classification algorithms. Consequently, the authors present an intuitive activity-based evaluation approach for adaptive web-based learning environments.

One of the possible dimensions of an e-Learning system is the adaptation to the knowledge level of the user. The authors in [12] extended an IEEE reference model for Learning Technology Systems Architecture (LTSA) [13] to include knowledge level adaptability. The proposed extension alongside the LTSA form an adaptable web-based environment that relies on the users behavior as one of the evaluation parameters.

Automatic evaluation of online learning assignments is discussed in [14] as the authors highlight its importance and applicability. The authors reflected on the promising outcomes of an automatic assessment tool, based on Latent Semantic Analysis, that has been examined in a specific online learning environment. Using automatic scoring of an assignment gives the learners an instant access to their performance anytime, without the need for direct supervision by an instructor. Such automation makes it more flexible for learners to schedule their time and to work on their weak points.

In [15] the authors focused on the learners' requirements to achieve a flexible web-based learning system. The proposed evaluation methods rely on introducing the semantic web and cognitive maps to the LTSA and Sharable Content Object Reference Model (SCORM) [16]. The analysis of semantic web content and the cognitive model of the students is discussed by different researchers in the learning and evaluation of learners knowledge [17]-[22]. For example, intelligent agents are supposed to enhance self assessment and feedbacks in eLearning environments that are based on concept maps [22].

\section{THE PROPOSED FramewORK}

The proposed framework for the automatic assessment of knowledge levels tries to consolidate the efforts of several researchers and standards [11]-[13], [16]. In addition, it includes the idea of mobile learning [2], [6] and flexible e-Learning environments. Furthermore, knowledge representation was inspired from the work of [17], [20], [22] which illustrates the role of "Concept Maps [20]" approach in learning and assessment. Fig. 1 represents an abstract architecture of the proposed e-Learning system.

The main aspects of the proposed framework are:

\section{A. User Experience}

The e-Learning users have the ability to access the educational system via different web-enabled means with disregard

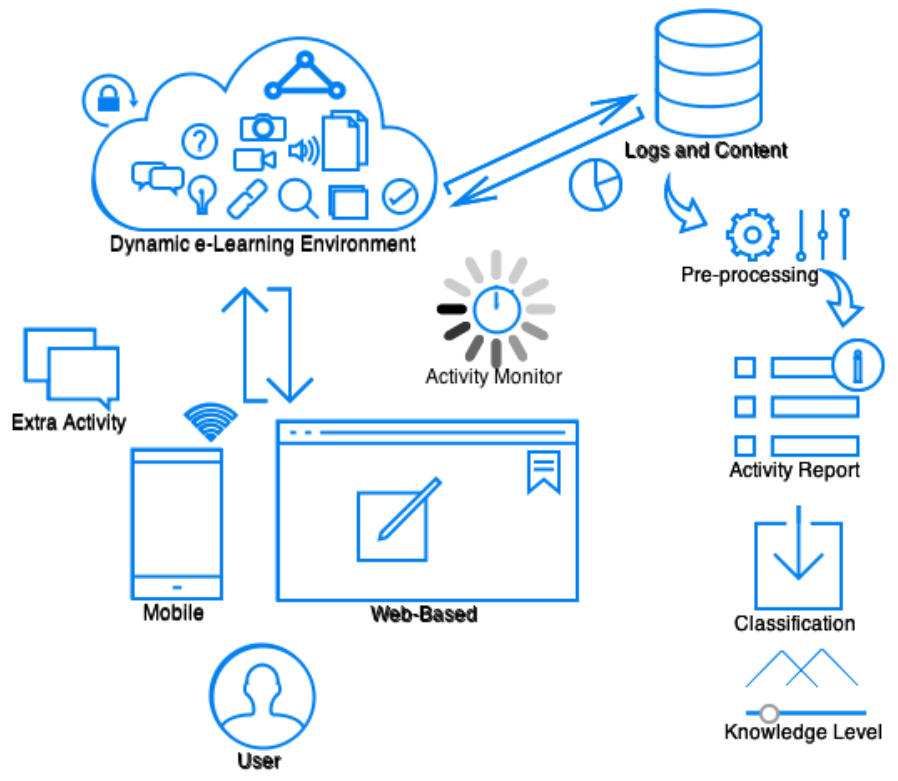

Fig. 1: Proposed e-Learning System Architecture

to time schedules. It is meant to be flexible and adaptable learning environment to the users' lifestyle. The objective of device-independent suitability is to offer a wide range of options to those unable to learn and work under strict time schedules, for example employees, housewives, travelers, and learners with high possibility of relocation. Further advantages include the opportunity of global reach or possible placeindependent collaboration between content providers.

The ultimate goal of any learning process is to deliver the proper amount of knowledge and to accurately judge the attainment of the goals. The authors in [23] investigated the possibility of tracking the user attention for higher level of engagement. In the absence of human control, tracking the user environment and providing a natural user interface would solve the reliability issue of such autonomous systems. Moreover, language teaching and practical training needs incorporating the users in a different manner, for example voice/motion recognition and evaluation.

\section{B. Cloud-Based Environment}

Cloud-based e-learning systems [24] offer a wide range of opportunities, for example competitive cost accompanied by a high level of scalability. The proposed secure cloud-based environment offers a number of services for rich user experience. Concurrently, there is a subset of services (Intelligent Agents) dedicated to record the user activities. The collection of the activity logs and the examination results of each user will be stored in a repository. Later on, the repository will be used in evaluating the knowledge level of the user.

Two main consideration are important here that are the design of the content and the delivery method. In [25] the authors highlighted one of the issues related to the acceptance of digital newspaper reading over paper-based version. Though, the delivery system of the digital content requires a significant value proposition in order to have higher rate of acceptance. 


\section{Incorporating Concept Maps}

Concept Maps have been highlighted recently by several researchers in the domain of automatic assessment of learners' knowledge [26]-[28]. In the theme of distance learning and self assessment, Concept Maps have various advantages over traditional approaches. The maps of the concepts will be an important tool in order to understand the level of knowledge for each system user.

The contribution is expected to highly facilitate a modern and efficient autonomous method of evaluation. A simple map consists of concepts denoted by circles in Fig. 2 while the underlying relations are denoted by annotated links. Also, it is possible to use concept maps in conjunction with rich media objects for a higher level of content enrichment.

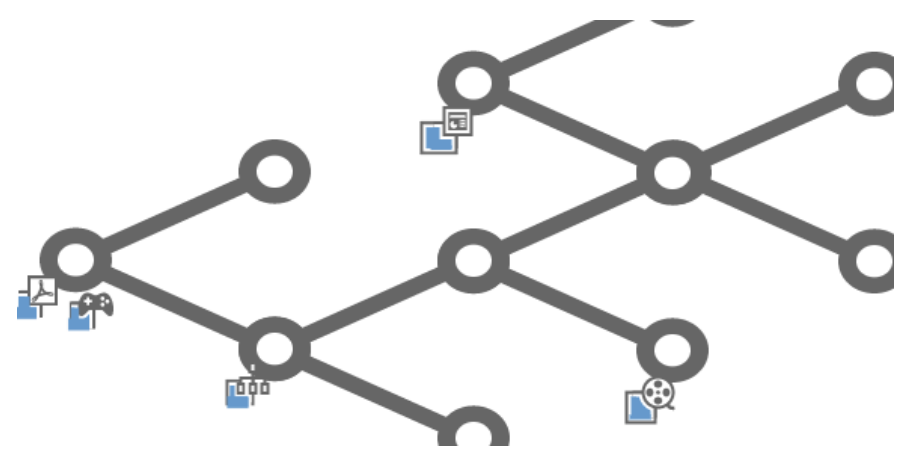

Fig. 2: Sample Concept Map

\section{Generating Activity Reports}

A summary of the user activity includes several derived variables that represent the activity, concept maps assessment, and examination results. Each variable is a result of a preprocessing step that produces a representative value of the corresponding activity. Part of the variables are to be produced by an approach similar to the work in [11]. Pre-processing step determines which specific attributes per user will be processed by the consecutive classification step. Main pre-processing aspects are as follows:

- Feature extraction and selection from the user activity log (for example average time spent per study objective).

- Categorizing the extracted features.

- Concept map analysis.

- Normalizing the results of the exam sets.

- $\quad$ Attention level (for example average time of focus).

This part of the system is essential and would need a thorough investigation in order to come up with a robust and efficient processing algorithms. One of the main concerns is to find linear and straightforward mappings between the inputs and the computed output values [29].

\section{E. Knowledge Level Assessment}

The ultimate goal of the proposed system is to provide an accurate and automatic evaluation of the knowledge level by analyzing the variables in the activity report. An effective Machine Learning method for classification provides the desired outcomes using the user activity model. However, there are various classification algorithms that vary in performance and require a solid experimental methodology for selecting the proper algorithm.

Two possible configurations need to be considered before building the classification models, in terms of domain knowledge dependence, to be either generic or specific. Proper selection of the configuration will result in a classifier for a specific domain, or alternatively a generic classifier. However, this research relies on a specific data set in a specific domain in building the classification models. On which several classification algorithms show promising results towards fulfilling the requirements of knowledge evaluation.

\section{DATA DESCRIPTION AND EVAluATION METRICS}

The machine learning classification models are built and evaluated in this research using the user knowledge modeling data set $^{1}$ for an e-Learning environment [11]. The data set represents the user's model and divided into three main sections that are the individual behavioral attributes, the exam performance attributes, and the knowledge level as target attribute. There are 403 instances from which 145 instances are used for model validation and the rest are used for creating the models. Table I shows the five input variables and the output variable description, Fig. 3 illustrates the distribution of the training instances, and Fig. 4 illustrates the distribution of the test instances.

\section{TABLE I: Input and Output Variables}

\begin{tabular}{cl} 
Variable & Description \\
\hline STG & The degree of study time for goal object materials \\
SCG & The degree of repetition number of user for goal object materials \\
STR & The degree of study time of user for related objects with goal object \\
LPR & The exam performance of user for related objects with goal object \\
PEG & The exam performance of user for goal objects \\
UNS & The knowledge level of user (Output Classes) \\
& Very Low (Beginner) \\
& Low (Intermediate) \\
& Middle (Expert) \\
& High (Advanced)
\end{tabular}

Several algorithms in this research attempted to classify the "test" instances using the developed model, where building the model is based on the "training" instances only. Fig. 5 illustrates the "Confusion or Contingency Matrix"; which is a visual representation of the classified (predicted) instances confronted with the actual instances [30]. The performance evaluation of the applied algorithms relies on different measures over the classified instances.

The classification's "confusion", alternatively called classification error, is illustrated in the rows holding actual classes and columns holding the predicted classes. The number of correctly classified instances resides diagonally in True Positive (TP) and True Negative (TN). TN is the "Actual Correct" classified as "True" while TN is the "Actual Incorrect" classified as "False". Better performing algorithm will have the highest number of TP and TN. Incorrectly classified will be in False Positive (FP) and False Negative (FN) which are "Actual

\footnotetext{
${ }^{1}$ https://archive.ics.uci.edu/ml/datasets/User+Knowledge+Modeling
} 


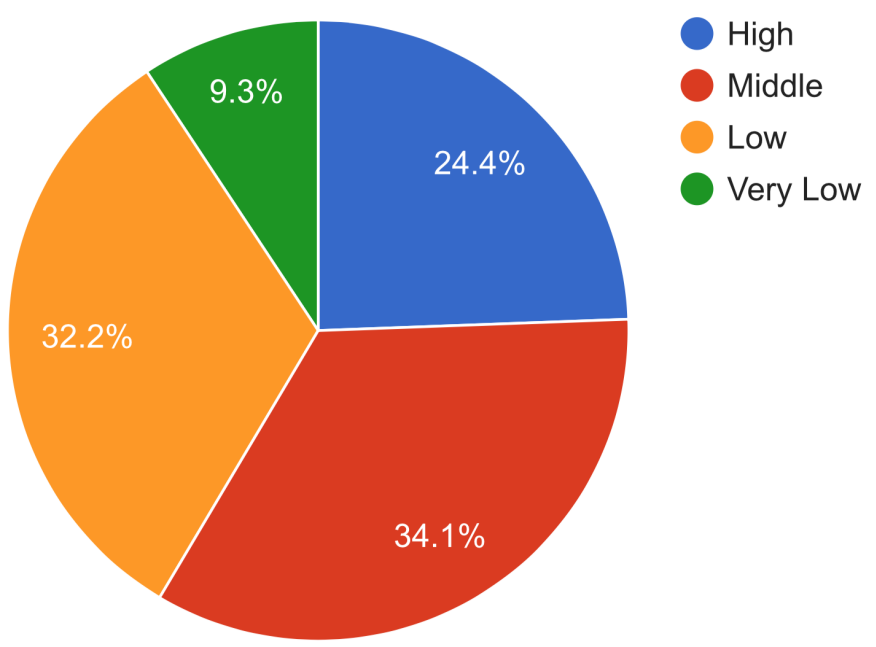

Fig. 3: Distribution of the training instances

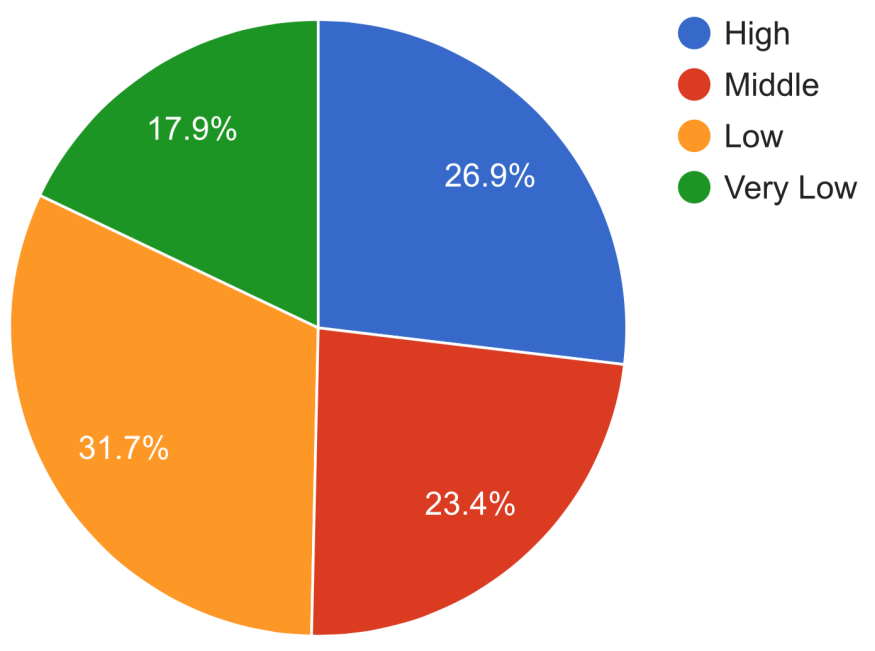

Fig. 4: Distribution of the test instances

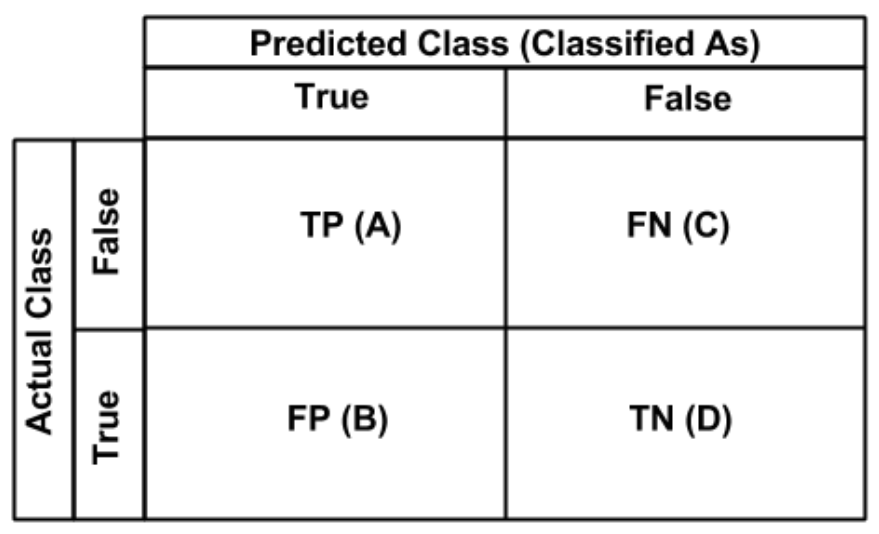

Fig. 5: Confusion Matrix
Correct" classified as "False" and "Actual Incorrect" classified as "True" respectively. The total number of the count in the all the cells (A, B, C, and D) is equal to the total number of the input instances.

Relying on the confusion matrix it is possible to formulate several performance indicators that give better understanding of the results. The empirical results of this research are used to report the following performance measure:

- Total Accuracy (Correctly Classified Instances)

$$
\text { TotalAccuracy }=\frac{T P+T N}{T P+T N+F P+F N}
$$

- Recall (alternatively TP Rate, Sensitivity, Hit Rate, or Type II Error)

$$
\text { Recall }=\frac{T P}{T P+F N}
$$

- Precision (Confidence or Type I Error)

$$
\text { Precision }=\frac{T P}{T P+F P}
$$

- F-Measure

$$
F \text {-Measure }=\frac{2 \times \text { Precision } \times \text { TPRate }}{\text { Precision } \times \text { TPRate }}
$$

- Area Under Receiver Operating Characteristics Curve (ROC) [31] [30] is a statistical measure that helps in comparing the performance of the classifiers. Such measure indicates the probability of a classification algorithm in giving higher rank for positive value over negative values, in other words it confronts the TP rate to the TN rate.

- $\quad$ Root Mean Squared Error (RMSE)

$$
R M S E=\sqrt{\sum_{i=1}^{n} \frac{\left(y_{i}-\hat{y}_{i}\right)^{2}}{n}}
$$

- $\quad$ Mean Absolute Error (MAE)

$$
M A E=\frac{1}{n} \sum_{i=1}^{n}\left|y_{i}-\hat{y}_{i}\right|
$$

- Relative Absolute Error (RAE)

$$
R A E=\sum_{i=1}^{n} \frac{\left|y_{i}-\hat{y}_{i}\right|}{y_{i}}
$$

- $\quad$ Root Relative Squared Error (RRSE)

$$
R R S E=\sqrt{\frac{\sum_{i=1}^{n}\left(y_{i}-\hat{y}_{i}\right)^{2}}{\sum_{i=1}^{n}\left(y_{i}-\bar{y}_{i}\right)^{2}}}
$$




\section{RESUlTS AND Discussion}

In this research, several experiments were made to analyze the performance of the selected classification algorithms in predicting the knowledge level of the e-learning user. The algorithms are J48 [32] , Random Forest (RF) [33], Multi Layer Perceptron Neural Network (MLP) [34], Simple Logistic (SL) [35], Bayes Network (BN) [36], [37], Naive Bayes (NB) [38], and Support Vector Machine (SVM) [39], [40].

The implemented algorithms create a model using the training data set and consequently use the test data set for validation. Table II shows the tuned parameters of the applied algorithms.

TABLE II: Tuned parameters of the algorithms

\begin{tabular}{cl} 
Algorithm & Parameters \\
\hline RF & $\begin{array}{l}\text { Number of trees to be built is 100 } \\
\text { with no restriction on the tree depth }\end{array}$ \\
MLP & $\begin{array}{l}\text { Four hidden layers, Sigmoid activation function, Learning Rate 0.3, } \\
\text { Momentum 0.2, and 500 Epochs }\end{array}$ \\
SL & Heuristic Stop 50, and Maximum Boosting Iterations are 500 \\
BN & Alpha of 0.5 for simple estimator \\
SVM & C-SVC Kernel, Gamma 0.069, and Cost 1000 (by GridSearch)
\end{tabular}

The classification results of the test data set illustrate a significant performance superiority of SVM, MLP, and SL in terms of total accuracy. Incorrectly classified instances are $1.39 \%$ of the total prediction results for the best models. Referring to total accuracy of all the models in Fig. 6, It is clear that Naive based algorithms are unable to exceed $88 \%$ of total accuracy, while Tree-based algorithms are slightly better.

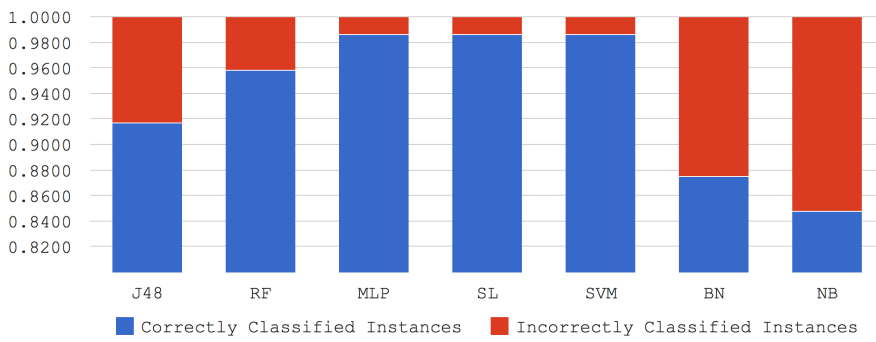

Fig. 6: Total accuracy of the built models over test dataset

Further analysis of the error rate is illustrated in Fig. 7, in which the SVM model demonstrates the lowest error margin for all the measures. Followed by MLP and SL respectively. Tree-based and Naive-based algorithms show several variations among the different measures, which makes it more difficult to judge which one has an overall better error margin.

The analysis of the confusion matrix for all the classes is summarized and illustrated in Fig. 8, in which the weighted average of all the measures for all the classes represents the overall performance of the algorithms.

Fig. 9, 10, 11, and 12 confront the classification results drawn from the confusion matrix for the four classes. Where the variations in the performance of each algorithm for the different classes is clear. Also it is apparent that MLP, SL, and SVM are less tolerant to the differences between the classes.

By looking at all the performance measures, it is evident that SVM-based classification models over-perform the other

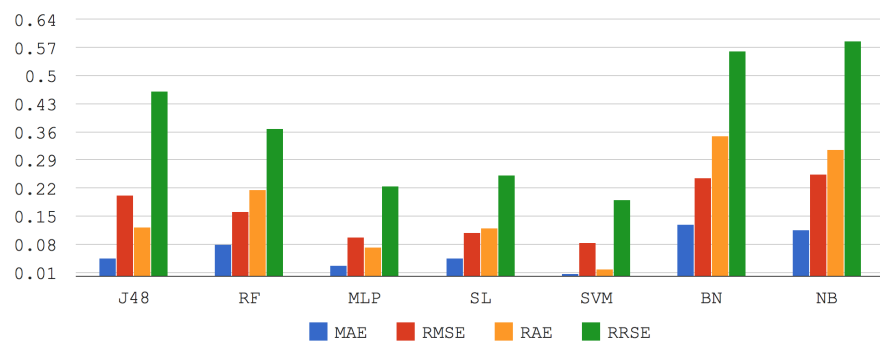

Fig. 7: Performance

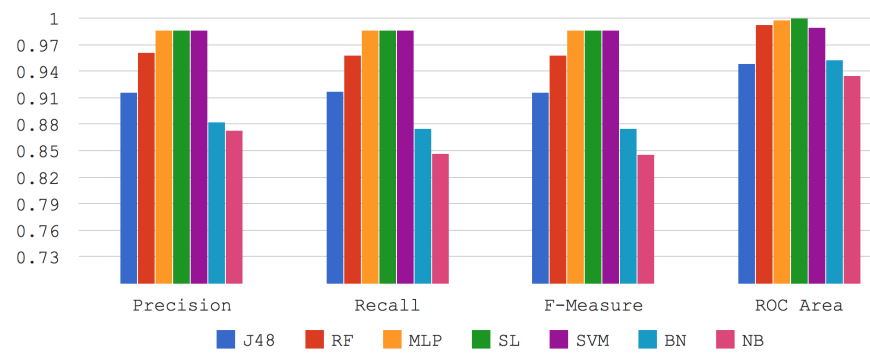

Fig. 8: Weighted average for all the classes

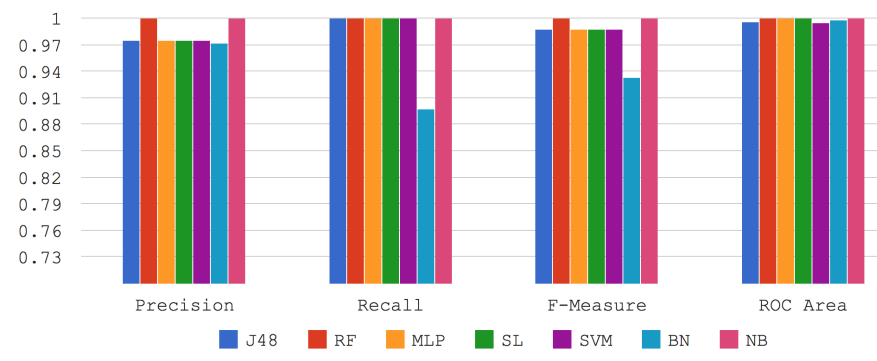

Fig. 9: High class

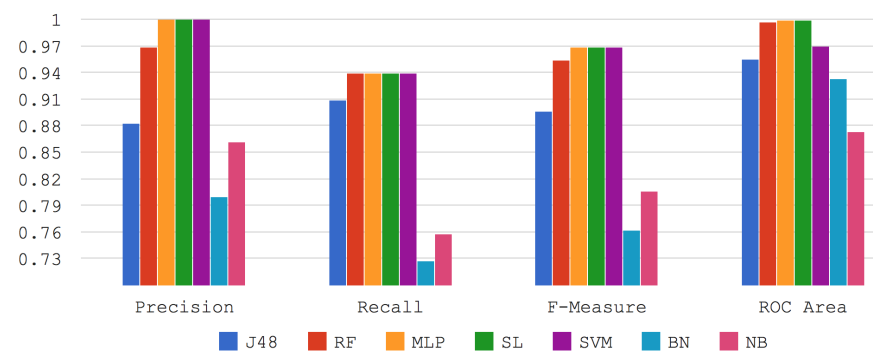

Fig. 10: Middle class

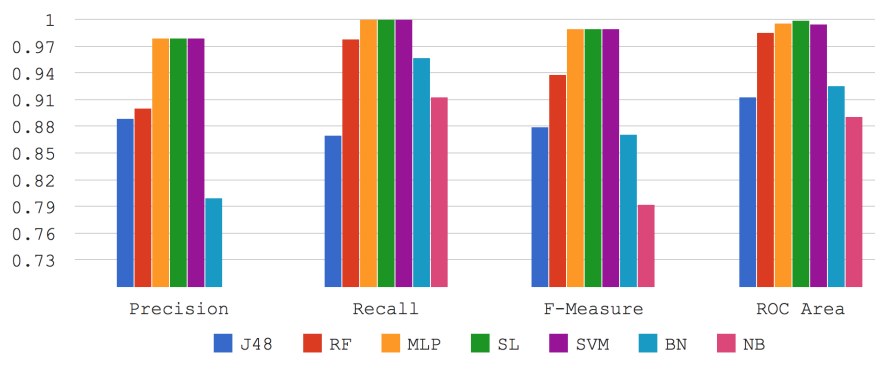

Fig. 11: Low class 


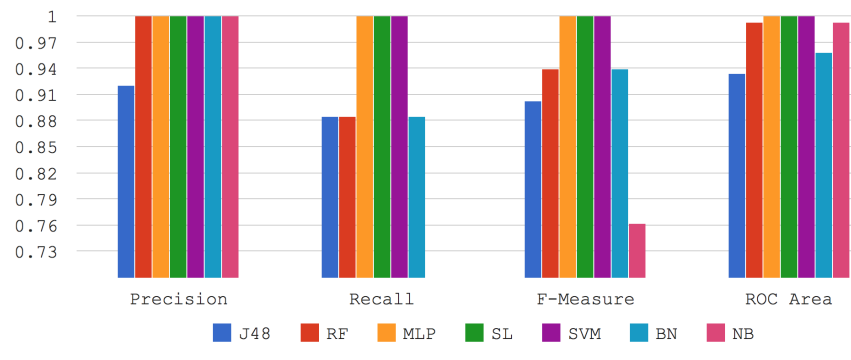

Fig. 12: Very Low class

machine learning approaches in this study, with disregard to ROC for SL. Moreover, SVM-based models show more stability among different classes and has the lowest error margin. According to this conclusion, the weighted average of precision, recall, f-measure and ROC are presented in Table III.

TABLE III: Weighted average of the performance measures

\begin{tabular}{ccccc} 
Algorithm & Precision & Recall & F-Measure & ROCArea \\
\hline SVM & 0.986 & 0.986 & 0.986 & 0.99 \\
SL & 0.986 & 0.986 & 0.986 & 1 \\
MLP & 0.986 & 0.986 & 0.986 & 0.998 \\
RF & 0.961 & 0.958 & 0.958 & 0.993 \\
J48 & 0.916 & 0.917 & 0.916 & 0.949 \\
BN & 0.883 & 0.875 & 0.875 & 0.953 \\
NB & 0.873 & 0.847 & 0.846 & 0.935
\end{tabular}

The predictions of the SVM-based model were highly accurate, however the algorithms seems to be clustered into three main groups. The first group has SVM, MLP, and SL; the second group includes Random Trees; and the third group includes Naive-based algorithms. Consequently, there is a relation between the general type of the classification algorithm and its performance.

\section{CONCLUSions AND Future WORK}

This research introduced a number of enhancements to dynamic e-Learning systems in terms of knowledge delivery and evaluation. It proposed an abstract framework of futuristic learning systems with more focus on the use of machine learning for evaluation. The analysis of various user activities in such dynamic environments leads to a comprehensive evaluation scheme, that is not biased and improves commonly used evaluation methods. In literature there exists a tremendous amount of attempts to empirically prove the accuracy of agentbased evaluation and knowledge dissemination over traditional approaches. Combining the research efforts found in literature and several standards led to the formulation of a common base for the prospective learning systems, at the same time acts as a technological enabler for the e-Learning trends. SVM models over-perform several classification models in terms of stability and error rates, therefore SVM-based models can play an important role in the evaluation of knowledge levels. MLP and SL models show a competitive performance, while Naive and Tree based models do not show a significant capability.

Future work needs to investigate the importance of input variables to the classification method. Possibly, importanceweighted input variables would increase the prediction accu- racy as it would mimic the evaluation behavior of humanbased methods. Dimensionality reduction and the tradeoff between domain knowledge independence and model generalization is an issue that is not covered in this research work. Furthermore, the adaptability of the delivered content to the users according to knowledge level in serving educational programs and training as well. It would be also possible to use the dynamic learning environment in corporate learning and workers evaluation. Looking further for a totally autonomous and adaptive e-Learning system in the future.

\section{ACKNOWLEDGMENT}

The author would like to acknowledge the efforts of Machine Learning Group at the University of Waikato for the development of Waikato Environment for Knowledge Analysis (WEKA).

\section{REFERENCES}

[1] M. J. Rosenberg, E-Learning: Strategies for Delivering Knowledge in the Digital Age. New York, NY, USA: McGraw-Hill, Inc., 2002.

[2] A. Fayyoumi, H. Mohammad, and H. Faris, "Mobile based learning and examination: Students and instructors perceptions from different arab countries," Journal of Software Engineering and Applications, vol. 6, no. 12, p. 662, 2013.

[3] A. Oztekin, D. Delen, A. Turkyilmaz, and S. Zaim, "A machine learning-based usability evaluation method for elearning systems," Decision Support Systems, vol. 56, pp. 63 - 73, 2013.

[4] S. S. Al-Gahtani, "Empirical investigation of e-learning acceptance and assimilation: A structural equation model," Applied Computing and Informatics, 2014.

[5] W. S. Chow and S. Shi, "Investigating students' satisfaction and continuance intention toward e-learning: An extension of the expectation confirmation model," Procedia - Social and Behavioral Sciences, vol. 141, pp. 1145 - 1149, 2014, 4th World Conference on Learning Teaching and Educational Leadership (WCLTA-2013), 27-29 October 2013, Barcelona, Spain.

[6] A. Zamfiroiu and C. Sbora, "Statistical analysis of the behavior for mobile e-learning," Procedia Economics and Finance, vol. 10, no. 0, pp. 237 - 243, 2014, international Conference on Applied Statistics, ICAS2013, 14-19 May 2013, Maha Sarakham, Thailand.

[7] A. Oztekin, Z. J. Kong, and O. Uysal, "Uselearn: A novel checklist and usability evaluation method for elearning systems by criticality metric analysis," International Journal of Industrial Ergonomics, vol. 40, no. 4, pp. $455-469,2010$.

[8] G. Mozhaeva, A. Feshchenko, and I. Kulikov, "E-learning in the evaluation of students and teachers: Lms or social networks?" Procedia - Social and Behavioral Sciences, vol. 152, pp. 127 - 130, 2014, international Congress on Education, ERPA Congress 2014, 6-8 June 2014, Istanbul, Turkey.

[9] S. Hubackova, "Evolution and evaluation of e-learning," Procedia - Social and Behavioral Sciences, vol. 171, pp. 231 - 235, 2015, 5th ICEEPSY International Conference on Education and Educational Psychology, 22-25 October 2014, Kyrenia, Cyprus.

[10] F. Castro, A. Vellido, A. Nebot, and F. Mugica, "Applying data mining techniques to e-learning problems," in Evolution of Teaching and Learning Paradigms in Intelligent Environment, ser. Studies in Computational Intelligence, L. Jain, R. Tedman, and D. Tedman, Eds. Springer Berlin Heidelberg, 2007, vol. 62, pp. 183-221.

[11] H. T. Kahraman, S. Sagiroglu, and I. Colak, "The development of intuitive knowledge classifier and the modeling of domain dependent data," Knowledge-Based Systems, vol. 37, pp. 283-295, Jan. 2013.

[12] P. Tzouveli, P. Mylonas, and S. Kollias, "An intelligent e-learning system based on learner profiling and learning resources adaptation," Computers and Education, vol. 51, no. 1, pp. 224-238, Aug. 2008.

[13] "Ieee standard for learning technology-learning technology systems architecture (ltsa)," IEEE Std 1484.1-2003, pp. 0_1-97, 2003. 
[14] M. Farrús and M. R. Costa-jussà, "Automatic evaluation for e-learning using latent semantic analysis: A use case," The International Review of Research in Open and Distributed Learning, vol. 14, no. 1, pp. 239254, 2013.

[15] A. Canales, A. Peña, R. Peredo, H. Sossa, and A. Gutiérrez, "Adaptive and intelligent web based education system: Towards an integral architecture and framework," Expert Systems with Applications, vol. 33, no. 4, pp. 1076-1089, Nov. 2007.

[16] J. Poltrack, N. Hruska, A. Johnson, and J. Haag, "The next generation of scorm: Innovation for the global force," in The Interservice/Industry Training, Simulation \& Education Conference (I/ITSEC), vol. 2012, no. 1, 2012.

[17] N. Ghatasheh, A. Najdawi, M. Abu-Faraj, and H. Faris, "Corporate elearning environment using concept maps: A case study," International Review on Computers and Software, vol. 8, no. 11, pp. 2655-2662, 2013.

[18] A. Najdawi and N. Ghatasheh, "Using concept mapping tools to enhance collaborative problem solving and innovation in corporate elearning," in Interactive Mobile and Computer Aided Learning (IMCL), 2012 International Conference on. Amman, Jordan: IEEE, 6-8 November 2012, pp. 197-199.

[19] B. Daley, C. Shaw, T. Balistrieri, K. Glasenapp, and L. Piacentine, "Concept maps: a strategy to teach and evaluate critical thinking," The Journal of nursing education, vol. 38, no. 1, pp. 42-47, January 1999.

[20] J. D. Novak and A. J. Cañas, "The theory underlying concept maps and how to construct them," Florida Institute for Human and Machine Cognition, vol. 1, 2006.

[21] L. Aroyo and D. Dicheva, "The new challenges for e-learning: The educational semantic web." Educational Technology \& Society, vol. 7, no. 4, pp. 59-69, 2004.

[22] A. Gladun, J. Rogushina, F. Garcı, R. Martínez-Béjar, J. T. FernándezBreis et al., "An application of intelligent techniques and semantic web technologies in e-learning environments," Expert Systems with Applications, vol. 36, no. 2, pp. 1922-1931, 2009.

[23] K. Liu, V. Tam, P. Tse, E. Lam, and V. Tam, "Developing the petal elearning platform for personalized teaching and learning," in Emerging Issues in Smart Learning, ser. Lecture Notes in Educational Technology, G. Chen, V. Kumar, Kinshuk, R. Huang, and S. C. Kong, Eds. Springer Berlin Heidelberg, 2015, pp. 119-122.

[24] B. Dong, Q. Zheng, J. Yang, H. Li, and M. Qiao, "An e-learning ecosystem based on cloud computing infrastructure," in Advanced Learning Technologies, 2009. ICALT 2009. Ninth IEEE International Conference on. Riga, Latvia: IEEE, 15-17 July 2009, pp. 125-127.

[25] L.-C. Fu, M.-H. Lu, H.-Y. Wu, W. Lin, and H.-P. Yueh, "College students? attitudes and preferences of mobile newspaper reading: A comparison between printed and web page layout," in Emerging Issues in Smart Learning, ser. Lecture Notes in Educational Technology, G. Chen, V. Kumar, Kinshuk, R. Huang, and S. C. Kong, Eds. Springer Berlin Heidelberg, 2015, pp. 105-106.

[26] A. Acharya and D. Sinha, "A weighted concept map approach to generate learning guidance in science courses," in Information Systems Design and Intelligent Applications, ser. Advances in Intelligent Systems and Computing, J. K. Mandal, S. C. Satapathy, M. Kumar Sanyal, P. P. Sarkar, and A. Mukhopadhyay, Eds. Springer India, 2015, vol. 340, pp. 143-152.

[27] X. Zhong, H. Fu, H. Xia, L. Yang, and M. Shang, "A hybrid cognitive assessment based on ontology knowledge map and skills," KnowledgeBased Systems, vol. 73, pp. 52-60, 2015.

[28] Y.-S. Lin, Y.-C. Chang, K.-H. Liew, and C.-P. Chu, "Effects of concept map extraction and a test-based diagnostic environment on learning achievement and learners' perceptions," British Journal of Educational Technology, 2015.

[29] M. Dixon, "A pattern oriented approach for designing scalable analytics applications (invited talk)," in Proceedings of the 2nd Workshop on Parallel Programming for Analytics Applications. San Francisco, California: ACM, 7-11 February 2015, pp. 4-8.

[30] D. M. W. Powers, "Evaluation: From Precision, Recall and F-Factor to ROC, Informedness, Markedness \& Correlation," School of Informatics and Engineering, Flinders University, Adelaide, Australia, Tech. Rep. SIE-07-001, 2007.
[31] T. Fawcett, "Roc graphs: Notes and practical considerations for researchers," HP Laboratories, Tech. Rep., 2004.

[32] S. Salzberg, "C4.5: Programs for machine learning by j. ross quinlan. morgan kaufmann publishers, inc., 1993," Machine Learning, vol. 16, no. 3, pp. 235-240, 1994.

[33] L. Breiman, "Random forests," Machine Learning, vol. 45, no. 1, pp. 5-32, 2001.

[34] S. Haykin, Neural Networks: A Comprehensive Foundation, 2nd ed. Upper Saddle River, NJ, USA: Prentice Hall PTR, 1998.

[35] M. Sumner, E. Frank, and M. Hall, "Speeding up logistic model tree induction," in Proceedings of the 9th European Conference on Principles and Practice of Knowledge Discovery in Databases, ser. PKDD’05. Porto, Portugal: Springer-Verlag, 3-7 October 2005, pp. 675-683.

[36] R. R. Bouckaert, Bayesian network classifiers in weka. Department of Computer Science, University of Waikato, 2004.

[37] S.-U. Normand and D. Tritchler, "Parameter updating in a bayes network," Journal of the American Statistical Association, vol. 87, no. 420, pp. 1109-1115, 1992.

[38] G. H. John and P. Langley, "Estimating continuous distributions in bayesian classifiers," in Proceedings of the Eleventh Conference on Uncertainty in Artificial Intelligence, ser. UAI'95. Montreal, Quebec, Canada: Morgan Kaufmann Publishers Inc., 18-20 August 1995, pp. 338-345.

[39] Y. El-Manzalawy and V. Honavar, "Wlsvm: integrating libsvm into weka environment," Software available at http://www. cs. iastate. edu/yasser/wlsvm, 2005.

[40] C.-C. Chang and C.-J. Lin, "Libsvm: a library for support vector machines," ACM Transactions on Intelligent Systems and Technology (TIST), vol. 2, no. 3, p. 27, 2011 